Hautarzt 2013 -64:552-552

DOI 10.1007/s00105-013-2539-8

Online publiziert: 12. August 2013

๑) Springer-Verlag Berlin Heidelberg 2013

C. Löser ${ }^{1}$ R. Kaufmann ${ }^{2}$

${ }^{1}$ Hautklinik, Hauttumorzentrum, Klinikum der Stadt Ludwigshafen am Rhein gGmbH, Ludwigshafen

${ }^{2}$ Klinik für Dermatologie, Venerologie und Allergologie, Universitätsklinikum Frankfurt, Frankfurt am Main

\title{
Faszination Dermatochirurgie
}

tet an Fachkollegen delegieren. Das ist Anspruch und Aufgabe des Dermatologen.

Dieses Leitthemenheft anlässlich der DGDC-Tagung in Tübingen präsentiert Übersichtsarbeiten namhafter Kollegen zu wichtigen Themen der Dermatochirurgie. Sie sollen einstimmen auf einen wichtigen Austausch. Und sie sollen aktuelle Fragen stellen und beantworten.

Löser, Zeymer und Nast besprechen den Umgang mit Blutverdünnern - ein wichtiges Reizthema, das von Ängsten und Mythen geprägt ist und in der Erstellung einer S3-Leitlinie durch DGDC und DDG eine evidenzbasierte Aufarbeitung erfahren soll. Mit seiner Übersicht über die „Chirurgische Therapie der epithelialen Hauttumore und ihrer Vorstufen" rekapituliert Häfner wesentliche Grundlagen der Dermatochirurgie. Darüber sollen ergänzende Techniken nicht in Vergessenheit geraten. Daher analysiert Bechara in der Arbeit „Sekundäre Wundheilung nach tumorchirurgischen Eingriffen im Kopf-Hals-Bereich" eine wichtige Alternative zum plastischen Wundverschluss. Die „Dermatochirurgische Therapie der Hyperhidrose" von Rapprich beschreibt das Spektrum der Behandlungsmöglichkeiten für eine Indikation, während Valesky, Kaufmann und Meissner in „Besondere Indikationen der Vakuumversiegelungstherapie in der Dermatochirurgie" verschiedene Einsatzmöglichkeiten für eine moderne Technik darstellen, die nicht nur bei der Wundbehandlung sinnvollen Einsatz findet. Mit der sachgerechten Behandlung von traumatischen und iatrogenen Verletzungsfolgen rundet Koller in "Chirurgische Therapie von Wunden und Narben" das Spektrum ab. Damit spannt sich ein Bogen von der Operationsvorbereitung bis zur Behandlung von Narben.
Während die Beiträge in diesem Heft gezielt die spezielle Dermatochirurgie behandeln, vertritt die DGDC auf dem Kongress in Tübingen natürlich auch die Ästhetik sowie die Belange des medizinischen Fachpersonals (www.dgdc.de).

Wir wünschen allen Lesern eine gewinnbringende Lektüre und empfehlen die Tagung als Forum für weiterführende Diskussionen, immer mit dem oben geschilderten Anspruch an den Dermatologen und mit Blick auf die uns anvertrauten Patienten.

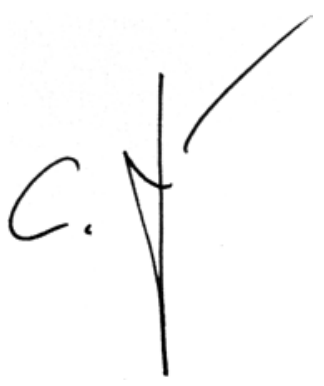

Dr. Christoph Löser

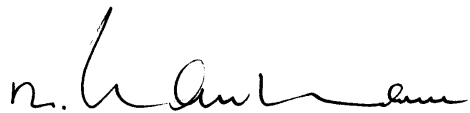

Prof. Dr. Roland Kaufmann

\section{Korrespondenzadressen}

\section{Dr. C. Löser}

Hautklinik, Hauttumorzentrum, Klinikum der Stadt Ludwigshafen am Rhein gGmbH Bremserstr. 79, 67063 Ludwigshafen loeserc@klilu.de

\section{Prof. Dr. R. Kaufmann}

Klinik für Dermatologie, Venerologie und Allergologie, Universitätsklinikum Frankfurt Theodor-Stern-Kai 7, 60590 Frankfurt am Main Kaufmann@em.uni-frankfurt.de 